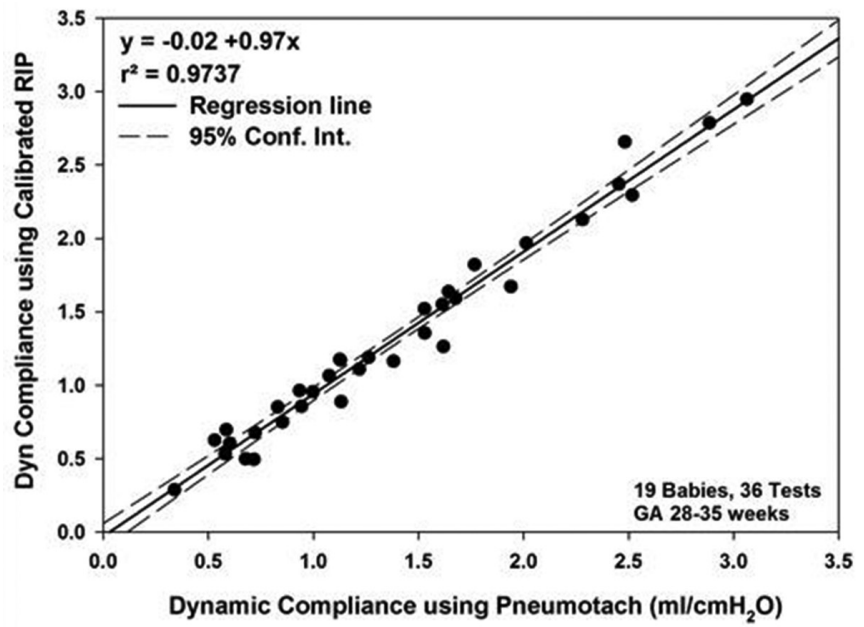

Abstract 0-077 Figure 1

linear regression algorithms on artefact-free breaths with amplitudes within the mean $\pm 1 S D$. A sequence of optimising iterations for computer breath selection produced best-fit regression coefficients with the PNT flow. Transpulmonary pressure was measured by esophageal catheter. Lung compliance and airway resistance were computed by a least mean square technique. Ribcage to abdominal phase angle (phi), Laboured breathing index (LBI), and phase relation in total breath (PhRTB) were computed from analysis of RIP ribcage and abdomen excursions. Validation measurements were performed on 18 infants of 28-35wks GA.

Results Correlation coefficients for compliance and resistance by PNT vs. RIP were $\mathrm{r}^{2}=0.9737$ and 0.8980 respectively. LBI for these infants was $1.2 \pm 0.6$, phi was $54.4 \pm 7.4$ degrees and PhRTB was $33.4 \pm 3.1 \%$.

Conclusion When properly calibrated, RIP derived respiratory mechanics measurements provide sufficient diagnostic accuracy in infants receiving NIV support.

\section{0-078 NEONATAL CHEST ULTRASOUND PREDICTS NON INVASIVE VENTILATION FAILURE IN PRETERM INFANTS}

F Raimondi, F Migliaro, A Sodano, C Coppola, S Lama, G Vallone, L Capasso. Translational Medical Sciences, Università "Federico II", Naples, Italy

10.1136/archdischild-2014-307384.145

Background Non invasive ventilation is the treatment of choice for neonatal moderate respiratory distress (RD). Predictors of nasal ventilation failure may be helpful in preventing clinical deterioration. Work on neonatal lung ultrasound has shown that the persistence of a hyperechogenic, "white lung" image correlates with severe distress in the preterm infant. In the present study we investigate the persistent white lung ultrasound image as a marker of non invasive ventilation failure.

Methods Newborns admitted to the Neonatal Intensive Care Unit with moderate RD and stabilised on nasal continuous positive airway pressure for 120 min were enrolled. Lung ultrasound was performed and blindly classified as Type 1 (white lung), Type 2 (prevalence of B-lines), or Type 3 (prevalence of A-lines). Chest radiograph was also examined and graded by an experienced radiologist blind to the infant's clinical condition. Main outcome of the study was the accuracy of bilateral Type 1 to predict intubation within $24 \mathrm{~h}$ from scanning. Secondary

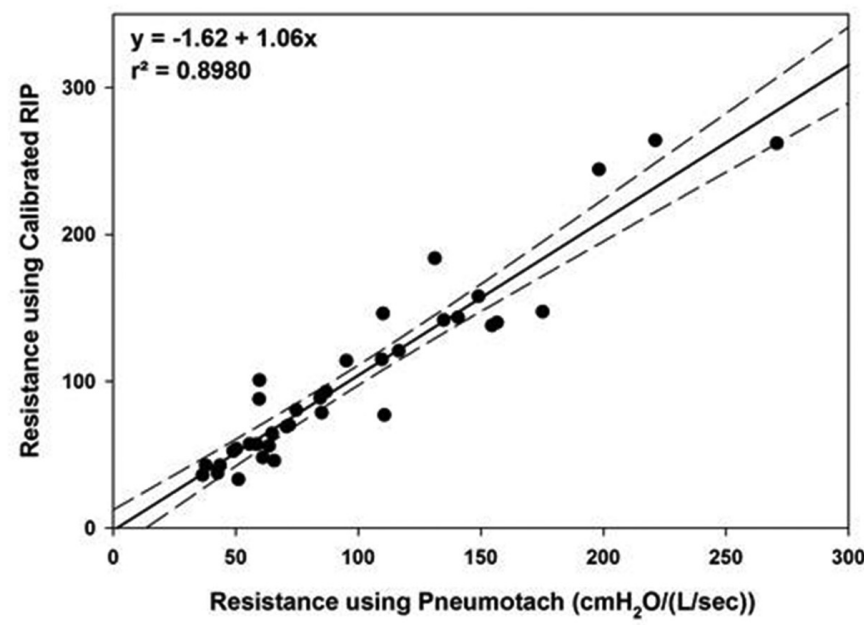

outcome was the performance of the highest radiographic grade within the same time interval.

Results Fifty-four preterm infants were enrolled (gestational age $32.5 \pm 2.6$ weeks; birthweight $1703 \pm 583$ grams). Type 1 lung profile showed sensitivity $88.9 \%$ (95\% CI 67.2-96.8), specificity 100\% (CI 94.9-100), PPV 100\% (CI 80.6-100), NPV 94.7\% (CI 82.7-98.5). Chest radiograph had sensitivity 38.9\% (95\% CI 20-61.1), specificity 77.8\% (CI 61.7-88.5), PPV 46.7\% (CI 24.8-69.9), NPV 71.8\% (CI 56.2-83.4).

Conclusions After a $2 \mathrm{~h}$ nasal ventilation trial, neonatal lung ultrasound is a useful predictor of the need for intubation, largely outperforming conventional radiology. Future studies should address whether including ultrasonography in the management of neonatal moderate RD confers clinical advantages.

\section{0-079 NASAL CONTINUOUS POSITIVE AIRWAY PRESSURE AND CARDIAC FUNCTION IN PRETERM INFANTS WITH LUNG DISEASE}

${ }^{1} \mathrm{~S}$ Rogerson, ${ }^{2} \mathrm{~F}$ Beker, ${ }^{1} \mathrm{P}$ Davis, ${ }^{3} \mathrm{~S}$ Hooper, ${ }^{4} \mathrm{~A}$ Sehgal. ${ }^{1}$ Neonatal Services, Royal Women's Hospital, Melbourne, Australia; ${ }^{2}$ Neonatal Services, Mercy Hospital for Women, Melbourne, Australia; ${ }^{3}$ Neonatal Services, Monash University, Melbourne, Australia; ${ }^{4}$ Neonatal Services, Monash Medical Cetre, Melbourne, Australia

\subsection{6/archdischild-2014-307384.146}

Introduction Previous studies of infants on ventilatory support suggest that right ventricular output (RVO) decreases with increasing mean airway pressure. This may be due to increased pressure in the thoracic cavity. We investigated changes in cardiac output $(\mathrm{CO})$ at different levels of nasal continuous positive airway pressure (nCPAP) in preterm infants with evolving chronic lung disease.

Methods We studied infants between 28 and 34 weeks corrected gestational age, a minimum of two weeks old, treated with nCPAP of 5 to $7 \mathrm{~cm} \mathrm{H} \mathrm{H}_{2} \mathrm{O}$, with an $\mathrm{O}_{2}$ requirement of $25-40 \%$, in whom written parental consent was obtained. Infants with significant cardiac shunts were excluded. Infants were randomly assigned to nCPAP levels of 4,6 , and $8 \mathrm{~cm} \mathrm{H}_{2} \mathrm{O}$ for $15 \mathrm{~min}$ each. Right and left ventricular output, left pulmonary artery flow, superior vena cava flow, heart rate and blood pressure were measured after each change with a Vivid-I ultrasound machine by a single examiner (FB) blinded to nCPAP levels. 